\title{
THE
}

$6-15-2000$

\section{Phase Changes in 38-Atom Lennard-Jones Clusters. I. A Parallel Tempering Study in the Canonical Ensemble}

\author{
J. P. Neirotti \\ University of Rhode Island \\ F. Calvo \\ David L. Freeman \\ University of Rhode Island, dfreeman@uri.edu \\ J. D. Doll
}

Follow this and additional works at: https://digitalcommons.uri.edu/chm_facpubs

Terms of Use

All rights reserved under copyright.

\section{Citation/Publisher Attribution}

Neirotti, J. P., Calvo, F., Freeman, D. L., \& Doll, J. D. (2000). Phase Changes in 38-Atom Lennard-Jones Cluster. I. A Parallel Tempering Study in the Canonicle Ensemble. Journal of Chemical Physics, 112(23), 10340-10349. doi: 10.1063/1.481671

Available at: http://dx.doi.org/10.1063/1.481671

This Article is brought to you for free and open access by the Chemistry at DigitalCommons@URI. It has been accepted for inclusion in Chemistry Faculty Publications by an authorized administrator of DigitalCommons@URI. For more information, please contact digitalcommons-group@uri.edu. 


\title{
Phase changes in 38-atom Lennard-Jones clusters. I. A parallel tempering study in the canonical ensemble
}

\author{
J. P. Neirotti \\ Department of Chemistry, University of Rhode Island, Kingston, Rhode Island 02881-0809 \\ F. Calvo \\ Département de Recherche sur la Matière Condensée, Service des Ions, Atomes et Agrégats, \\ CEA Grenoble, F38054 Grenoble Cedex, France \\ David L. Freeman \\ Department of Chemistry, University of Rhode Island, Kingston, Rhode Island 02881-0809 \\ J. D. Doll \\ Department of Chemistry, Brown University, Providence, Rhode Island 02912
}

(Received 27 January 2000; accpeted 23 March 2000)

\begin{abstract}
The heat capacity and isomer distributions of the 38-atom Lennard-Jones cluster have been calculated in the canonical ensemble using parallel tempering Monte Carlo methods. A distinct region of temperature is identified that corresponds to equilibrium between the global minimum structure and the icosahedral basin of structures. This region of temperatures occurs below the melting peak of the heat capacity and is accompanied by a peak in the derivative of the heat capacity with temperature. Parallel tempering is shown to introduce correlations between results at different temperatures. A discussion is given that compares parallel tempering with other related approaches that ensure ergodic simulations. (C) 2000 American Institute of Physics. [S0021-9606(00)51223-9]
\end{abstract}

\section{INTRODUCTION}

Because the properties of molecular aggregates impact diverse areas ranging from nucleation and condensation ${ }^{1}$ to heterogeneous catalysis, the study of clusters has continued to be an important part of modern condensed matter science. Clusters can be viewed as an intermediate phase of matter, and clusters can provide information about the transformation from finite to bulk behavior. Furthermore, the potential surfaces of clusters can be complex, and many clusters are useful prototypes for studying other systems having complex phenomenology.

The properties of small clusters can be unusual owing to the dominance of surface rather than bulk atoms. A particularly important and well studied example of a property that owes its behavior to the presence of large numbers of surface atoms is cluster structure. ${ }^{2-4}$ The structure of clusters can differ significantly from the structure of the corresponding bulk material, and these differences in structure have implications about the properties of the clusters. For example, most small Lennard-Jones (LJ) clusters have global potential surface minima that are based on icosahedral growth patterns. The fivefold symmetries of these clusters differ substantially from the closest-packed arrangements observed in bulk materials.

While most small Lennard-Jones clusters have geometries based on icosahedral core structures, there can be exceptions. ${ }^{2,5-7}$ A notable example is the 38-atom LennardJones cluster $\left[\mathrm{LJ}_{38}\right]$. This cluster is particularly interesting owing to its complex potential surface and associated phenomenology. The potential surface for $\mathrm{LJ}_{38}$ has been described in detail by Doye, Miller, and Wales ${ }^{5}$ who have care- fully constructed the disconnectivity graph ${ }^{8,9}$ for the system using information garnered from basin hopping and eigenvector following studies of the low energy potential minima along with examinations of the transition state barriers. The general structure of this potential surface can be imagined to be two basins of similar energies separated by a large energy barrier with the lowest energy basin being significantly narrower than the second basin. Striking is the global minimum energy structure for $\mathrm{LJ}_{38}$ which, unlike the case for most small Lennard-Jones clusters, is not based on an icosahedral core, but rather is a symmetric truncated octahedron. The vertices defined by the surface atoms of $\mathrm{LJ}_{38}$ have a morphology identical to the first Brillouin zone of a face centered cubic lattice, ${ }^{10}$ and the high symmetry of the cluster may account for its stability. It is interesting to note that recent experimental studies ${ }^{11}$ of nickel clusters using nitrogen uptake measurements have found the global minimum of $\mathrm{Ni}_{38}$ to be a truncated octahedron as well. The basin of energy minima about the global minimum of $\mathrm{LJ}_{38}$ is narrow compared to the basin about the next highest energy isomer which does have an icosahedral core. The difference in energy between the global minimum and the lowest minimum in the icosahedral basin is only $0.38 \%$ of the energy of the global minimum. ${ }^{5}$

Characteristic of some thermodynamic properties of small clusters are ranges of temperature over which these properties change rapidly in a fashion reminiscent of the divergent behavior known to occur in bulk phase transitions at a single temperature. The rapid changes in such thermodynamic properties for clusters are not divergent and occur over a range of temperatures owing to the finite sizes of the systems. In accord with the usage introduced by Berry, Beck, 
Davis, and Jellinek ${ }^{12}$ we refer to the temperature ranges where rapid changes occur as "phase change" regions, rather than using the term "phase transition," that is reserved for systems at the thermodynamic limit. As an example $\mathrm{LJ}_{55}$ displays a heat capacity anomaly over a range in temperatures often associated with what has been termed "cluster melting." 13 Molecular dynamics and microcanonical simulations performed at kinetic temperatures in the melting region of $\mathrm{LJ}_{55}$ exhibit van der Waals type loops in the caloric curves and coexistence between solidlike and liquidlike forms.

In recent studies, Doye, Wales, and Miller ${ }^{14}$ and Miller, Doye, and Wales ${ }^{15}$ have examined the phase change behavior of $\mathrm{LJ}_{38}$. These authors have calculated the heat capacity and isomer distributions as a function of temperature using the superposition method. ${ }^{16,17}$ In the superposition method the microcanonical density of states is calculated for each potential minimum, and the total density of states is then constructed by summation with respect to each local density of states. Because it is not possible to find all potential minima for a system as complex as $\mathrm{LJ}_{38}$, the summation is augmented with factors that represent the effective weights of the potential minima that are included in the sum. The superposition method has also been improved to account for anharmonicities and stationary points. ${ }^{17}$ For $\mathrm{LJ}_{38}$ Doye et al. ${ }^{14}$ have identified two phase change regions. The first, accompanied by a heat capacity maximum, is associated with a solid-to-solid phase change between the truncated octahedral basin and the icosahedral basin. A higher temperature heat capacity anomaly represents the solid-liquid coexistence region, similar to that found in other cluster systems. The heat capacity anomaly associated with the melting transition in $\mathrm{LJ}_{38}$ is steeper and more pronounced than the heat capacity peak that Doye et al. ${ }^{14}$ have associated with the solid-solid transition. Because the weights that enter in the sum to construct the microcanonical density of states are estimated, it is important to confirm the findings of Doye et al. ${ }^{14}$ by detailed numerical simulation. Such simulations are a goal of the current work and its companion paper. As is found in Sec. III, the simulations provide a heat capacity curve for $\mathrm{LJ}_{38}$ that has some qualitative differences with the curve reported by Doye et al. ${ }^{14}$

Owing to the complex structure of the potential surface of $\mathrm{LJ}_{38}$, the system represents a particularly challenging case for simulation. It is well known that simulations of systems having more than one important region of space separated by significant energy barriers can be difficult. The difficulties are particularly severe if any of the regions are either narrow or reachable only via narrow channels. The narrow basin about the global minimum makes simulations of $\mathrm{LJ}_{38}$ especially difficult. There are several methods that have been developed that can prove to be useful in overcoming such ergodicity difficulties in simulations. Many of these methods use information about the underlying potential surface generated from simulations on the system using parameters where the various regions of configuration space are wellconnected. One of the earliest of these methods is $\mathrm{J}$-walking ${ }^{18}$ where information about the potential surface is obtained from simulations at high temperatures, and the in- formation is passed to low temperature walks by jumping periodically to the high temperature walk. Closely allied with J-walking is the parallel tempering method ${ }^{19-23}$ where configurations are exchanged between walkers running at two differing temperatures. A related approach, ${ }^{24}$ similar in spirit to J-walking, uses Tsallis distributions that are sufficiently broad to cover much of configuration space. Another recent addition $^{25}$ to these methods is the use of multicanonical distributions ${ }^{26}$ in the jumping process. Multicanonical walks are performed using the entropy of the system, and multicanonical distributions are nearly independent of the energy thereby allowing easy transitions between energy basins. As we discuss in the current work, we have found the parallel tempering method to be most useful in the context of simulations of $\mathrm{LJ}_{38}$. A comparative discussion of some of the methods outlined above is given later in this paper.

In the current work we apply parallel tempering to the calculation of the thermodynamic properties of $\mathrm{LJ}_{38}$ in the canonical ensemble. In the paper that follows ${ }^{27}$ we again use parallel tempering to study $\mathrm{LJ}_{38}$, but using molecular dynamics methods along with microcanonical Monte Carlo simulations. Our goals are to understand better this complex system and to determine the best simulation method for systems of comparable complexity. The contents of the remainder of this first paper are as follows. In Sec. II we discuss the methods used with particular emphasis on the parallel tempering approach and its relation to the J-walking method. In Sec. III we present the results including the heat capacity as a function of temperature and identify the phase change behaviors of $\mathrm{LJ}_{38}$. In Sec. IV we present our conclusions and describe our experiences with alternatives to parallel tempering for insuring ergodicity.

\section{METHOD}

For canonical simulations we model a cluster with $N$ atoms by the standard Lennard-Jones potential augmented by a constraining potential $U_{c}$ used to define the cluster

$$
U(\mathbf{r})=4 \varepsilon \sum_{i<j}^{N}\left[\left(\frac{\sigma}{r_{i j}}\right)^{12}-\left(\frac{\sigma}{r_{i j}}\right)^{6}\right]+U_{c},
$$

where $\sigma$ and $\varepsilon$ are, respectively, the standard Lennard-Jones length and energy parameters, and $r_{i j}$ is the distance between particles $i$ and $j$. The constraining potential is necessary because clusters at defined temperatures have finite vapor pressures, and the evaporation events can make the association of any atom with the cluster ambiguous. For classical Monte Carlo simulations, a perfectly reflecting constraining potential is most convenient

$$
U_{c}=\sum_{i=1}^{N} u\left(\mathbf{r}_{i}\right),
$$

with

$$
u(\mathbf{r})=\left\{\begin{array}{ll}
\infty & \left|\mathbf{r}-\mathbf{r}_{\mathrm{cm}}\right|>R_{c} \\
0 & \left|\mathbf{r}-\mathbf{r}_{\mathrm{cm}}\right|<R_{c}
\end{array},\right.
$$

where $\mathbf{r}_{\mathrm{cm}}$ is the center of mass of the cluster, and we call $R_{c}$ the constraining radius. 
Thermodynamic properties of the system are calculated with Monte Carlo methods using the parallel tempering technique. ${ }^{19-23}$ To understand the application of the parallel tempering method and to understand the comparison of parallel tempering with other related methods, it is useful to review the basic principles of Monte Carlo simulations.

In the canonical ensemble the goal is the calculation of canonical expectation values. For example, the average potential energy is expressed

$$
\langle U\rangle=\frac{\int d^{3 N} r U(\mathbf{r}) e^{-\beta U(\mathbf{r})}}{\int d^{3 N} r e^{-\beta U(\mathbf{r})}},
$$

where $\beta=1 / k_{B} T$ with $T$ the temperature and $k_{B}$ the Boltzmann constant. In Monte Carlo simulations such canonical averages are determined by executing a random walk in configuration space so that the walker visits points in space with a probability proportional to the canonical density $\rho(\mathbf{r})$ $=Z^{-1} \exp [-\beta U(\mathbf{r})]$, where $Z$ is the configurational integral that normalizes the density. After generating $M$ such configurations in a random walk, the expectation value of the potential energy is approximated by

$$
\langle U\rangle_{M}=\frac{1}{M} \sum_{i=1}^{M} U\left(\mathbf{r}_{i}\right) .
$$

The approximate expectation value $\langle U\rangle_{M}$ becomes exact in the limit that $M \rightarrow \infty$.

A sufficiency condition for the random walk to visit configuration space with a probability proportional to the density $\rho(\mathbf{r})$ is the detailed balance condition ${ }^{28,29}$

$$
\rho\left(\mathbf{r}_{0}\right) K\left(\mathbf{r}_{0} \rightarrow \mathbf{r}_{n}\right)=\rho\left(\mathbf{r}_{n}\right) K\left(\mathbf{r}_{n} \rightarrow \mathbf{r}_{0}\right),
$$

where $\mathbf{r}_{0}$ and $\mathbf{r}_{n}$ represent two configurations of the system and $K\left(\mathbf{r}_{0} \rightarrow \mathbf{r}_{n}\right)$ is the conditional probability that if the system is at configuration $\mathbf{r}_{0}$ it makes a transition to $\mathbf{r}_{n}$. In many Monte Carlo approaches, the conditional probability is not known and is replaced by the expression

$$
K\left(\mathbf{r}_{0} \rightarrow \mathbf{r}_{n}\right)=T\left(\mathbf{r}_{0} \rightarrow \mathbf{r}_{n}\right) \operatorname{acc}\left(\mathbf{r}_{0} \rightarrow \mathbf{r}_{n}\right),
$$

where $T\left(\mathbf{r}_{0} \rightarrow \mathbf{r}_{n}\right)$ is called the trial probability and $\operatorname{acc}\left(\mathbf{r}_{0}\right.$ $\left.\rightarrow \mathbf{r}_{n}\right)$ is an acceptance probability constructed to ensure $K\left(\mathbf{r}_{0} \rightarrow \mathbf{r}_{n}\right)$ satisfies the detailed balance condition. The trial probability can be any normalized density function chosen for convenience. A common choice for the acceptance probability is given by ${ }^{28,29}$

$$
\operatorname{acc}\left(\mathbf{r}_{0} \rightarrow \mathbf{r}_{n}\right)=\min \left[1, \frac{\rho\left(\mathbf{r}_{n}\right) T\left(\mathbf{r}_{n} \rightarrow \mathbf{r}_{0}\right)}{\rho\left(\mathbf{r}_{0}\right) T\left(\mathbf{r}_{0} \rightarrow \mathbf{r}_{n}\right)}\right] .
$$

The Metropolis method, ${ }^{30}$ obtained from Eq. (8) by choosing $T\left(\mathbf{r}_{0} \rightarrow \mathbf{r}_{n}\right)$ to be a uniform distribution of points of width $\Delta$ centered about $\mathbf{r}_{0}$, is arguably the most widely used Monte Carlo method and the basis for all the approaches discussed in the current work. The Metropolis method rigorously guarantees a random walk visits configuration space proportional to a given density function asymptotically in the limit of an infinite number of steps. In practice when configuration space is divided into important regions separated by significant energy barriers, a low temperature finite Metropolis walk can have prohibitively long equilibration times.
Such problems in attaining ergodicity in the walk do not occur at temperatures sufficiently high that the system has significant probability of finding itself in the barrier regions. In both the J-walking and parallel tempering methods, information obtained from an ergodic Metropolis walk at high temperatures is passed to a low temperature walker periodically to enable the low temperature walker to overcome the barriers between separated regions. In the J-walking method $^{18}$ the trial probability at inverse temperature $\beta$ is taken to be a high temperature Boltzmann distribution

$$
T\left(\mathbf{r}_{0} \rightarrow \mathbf{r}_{n}\right)=Z^{-1} e^{-\beta_{J} U\left(\mathbf{r}_{n}\right)},
$$

where $\beta_{J}$ represents the jumping temperature that is sufficiently high that a Metropolis walk can be assumed to be ergodic. Introduction of Eq. (9) into Eq. (8) results in the acceptance probability

$$
\operatorname{acc}\left(\mathbf{r}_{0} \rightarrow \mathbf{r}_{n}\right)=\min \left\{1, \exp \left[-\left(\beta-\beta_{J}\right)\left(U\left(\mathbf{r}_{n}\right)-U\left(\mathbf{r}_{0}\right)\right)\right]\right\} .
$$

In practice at inverse temperature $\beta$ the trial moves are taken from the Metropolis distribution about $90 \%$ of the time with jumps attempted using Eq. (9) about $10 \%$ of the time. The jumping configurations are generated with a Metropolis walk at inverse temperature $\beta_{J}$, and jump attempts are accepted using Eq. (10). The acceptance expression [Eq. (10)] is correct provided the configurations chosen for jumping are a random representation of the distribution $e^{-\beta_{J} U(\mathbf{r})}$. The Metropolis walk that is used to generate the configurations at inverse temperature $\beta_{J}$ is correlated, ${ }^{28}$ and Eq. (10) is inappropriate unless jumps are attempted sufficiently infrequently to break the correlations. In practice Metropolis walks are still correlated after 10 steps, and it is not possible to use Eq. (10) correctly if jumps are attempted $10 \%$ of the time. In J-walking the difficulty with correlations is overcome in two ways. In the first method, often called serial J-walking, ${ }^{18}$ a large set of configurations is stored to an external distribution with the configurations generated with a Metropolis walk at inverse temperature $\beta_{J}$, and configurations are stored only after sufficient steps to break the correlations in the Metropolis walk. Additionally, the configurations are chosen from the external distribution at random. This external distribution is made sufficiently large that the probability of ever choosing the same configuration more than once is small. In this method detailed balance is strictly satisfied only in the limit that the external distribution is of infinite size. In the second method, often called parallel J-walking, ${ }^{31,32}$ the walks at each temperature are made in tandem on a parallel machine. Many processors, randomly initialized, are assigned to the jumping temperature, and each processor at the jumping temperature is used to donate a high temperature configuration to the low temperature walk sufficiently infrequently that the correlations in the Metropolis walk at inverse temperature $\beta_{J}$ are broken. In this parallel method, configurations are never reused, but the acceptance criterion [Eq. (10)] is strictly valid only in the limit of an infinite set of processors at inverse temperature $\beta_{J}$. In practice both serial and parallel J-walking work well for many applications with finite external distributions or with a finite set of processors. ${ }^{18,31-38}$ 
In parallel tempering ${ }^{19-23}$ configurations from a high temperature walk are also used to make a low temperature walk ergodic. In contrast to J-walking rather than the high temperature walk feeding configurations to the low temperature walk, the high and low temperature walkers exchange configurations. By exchanging configurations detailed balance is satisfied, once the Metropolis walks at the two temperatures are sufficiently long to be in the asymptotic region. To verify detailed balance is satisfied by the parallel tempering procedure we let

$$
\rho_{2}\left(\mathbf{r}, \mathbf{r}^{\prime}\right)=Z^{-1} e^{-\beta U(\mathbf{r})} e^{-\beta_{J} U\left(\mathbf{r}^{\prime}\right)}
$$

be the joint density that the low temperature walker is at configuration $\mathbf{r}$ and the high temperature walker is at configuration $\mathbf{r}^{\prime}$. When configurations between the two walkers are exchanged, the detailed balance condition is

$\rho_{2}\left(\mathbf{r}, \mathbf{r}^{\prime}\right) K\left(\mathbf{r} \rightarrow \mathbf{r}^{\prime}, \mathbf{r}^{\prime} \rightarrow \mathbf{r}\right)=\rho_{2}\left(\mathbf{r}^{\prime}, \mathbf{r}\right) K\left(\mathbf{r}^{\prime} \rightarrow \mathbf{r}, \mathbf{r} \rightarrow \mathbf{r}^{\prime}\right)$.

By solving for the ratio of the conditional transition probabilities

$\frac{K\left(\mathbf{r} \rightarrow \mathbf{r}^{\prime}, \mathbf{r}^{\prime} \rightarrow \mathbf{r}\right)}{K\left(\mathbf{r}^{\prime} \rightarrow \mathbf{r}, \mathbf{r} \rightarrow \mathbf{r}^{\prime}\right)}=\exp \left[-\left(\beta-\beta_{J}\right)\left(U\left(\mathbf{r}^{\prime}\right)-U(\mathbf{r})\right)\right]$,

it is evident that if exchanges are accepted with the same probability as the acceptance criterion used in J-walking [see Eq. (10)], detailed balance is satisfied.

Although the basic notions used by both J-walking and parallel tempering are similar, the organization of a parallel tempering calculation can be significantly simpler than the organization of a J-walking calculation. In parallel tempering no external distributions are required nor are multiple processors required at any temperature. Parallel tempering can be organized in the same simple way that serial tandem $\mathrm{J}$-walking is organized as discussed in the original J-walking reference. ${ }^{18}$ Unlike serial tandem J-walking where detailed balance can be attained only asymptotically, parallel tempering satisfies detailed balance directly. For a problem as difficult as $\mathrm{LJ}_{38}$ where very long simulations are required, the huge external distributions needed in serial J-walking, or the large set of jumping processors needed in parallel J-walking, make the method prohibitive. As discussed in Sec. III, parallel tempering can be executed for arbitrarily long simulations making the method suitable at least for $\mathrm{LJ}_{38}$.

In the current calculation parallel tempering is used not just to simulate the system at some low temperature using high temperature information, but simulations are performed for a series of temperatures. As is the case for J-walking ${ }^{18}$ and as discussed elsewhere for parallel tempering, ${ }^{22}$ the gaps between adjacent temperatures cannot be chosen arbitrarily. Temperature gaps must be chosen so that exchanges are accepted with sufficient frequency. If the temperature gap is too large, the configurations important at the two exchanging temperatures can be sufficiently dissimilar that no exchanges are ever accepted. Preliminary calculations must be performed to explore the temperature differences needed for acceptable exchange probabilities. In practice we have found at least $10 \%$ of attempted exchanges need to be accepted for the parallel tempering procedure to be useful. In general the temperature gaps must be decreased near phase change regions or when the temperature becomes low.

By exchanging configurations between temperatures, correlations are introduced at different temperature points. For example, the average heat capacities at two temperatures may rise or fall together as each value fluctuates statistically. In some cases the values of the heat capacities or other properties at two temperatures can be anticorrelated. The magnitude of these correlations between temperatures are measured and discussed in Sec. III. As discussed in Sec. III the correlations between differing temperatures imply that the statistical fluctuations must be sufficiently low to ensure any features observed in a calculation as a function of temperature are meaningful.

\section{RESULTS}

Forty distinct temperatures have been used in the parallel tempering simulations of $\mathrm{LJ}_{38}$ ranging from $T=0.0143 \varepsilon / k_{B}$ to $T=0.337 \varepsilon / k_{B}$. The simulations have been initiated from random configurations of the 38 atoms within a constraining sphere of radius $2.25 \sigma$. We have chosen $R_{c}=2.25 \sigma$, because we have had difficulties attaining ergodicity with larger constraining radii. With large constraining radii, the system has a significant boiling region at temperatures not far from the melting region, and it is difficult to execute an ergodic walk with any method when there is coexistence between liquidlike and vapor regions. Constraining radii smaller than $2.25 \sigma$ can induce significant changes in thermodynamic properties below the temperature of the melting peak. Using the randomly initialized configurations the initialization time to reach the asymptotic region in the Monte Carlo walk has been found to be long with about 95 million Metropolis Monte Carlo points followed by 190 million parallel tempering Monte Carlo points included in the walk prior to data accumulation. This long initiation period can be made significantly shorter by initializing each temperature with the structure of the global minimum. We have chosen to initialize the system with random configurations to verify the parallel tempering method is able to equilibrate this system with no prior knowledge about the structure of the potential surface. Following this initiation period, $1.3 \times 10^{10}$ points have been included with data accumulation. Parallel tempering exchanges have been attempted every 10 Monte Carlo passes over the 38 atoms in the cluster.

In an attempt to minimize the correlations in the data at differing temperatures, an exchange strategy has been used that includes exchanges between several temperatures. To understand this strategy, we let the set of temperatures be put into an array. One-half of the exchanges have been attempted between adjacent temperatures in the array, one-fourth have been attempted between next near neighboring temperatures, one-eighth between every third temperature, one-sixteenth between every fourth temperature, and one-thirty second between every fifth temperature in the array. We have truncated this procedure at the fifth near neighboring temperatures, because exchanges between temperatures differing by more than fifth neighbors are accepted with frequencies of less than $10 \%$. The data presented in this work have been gener- 


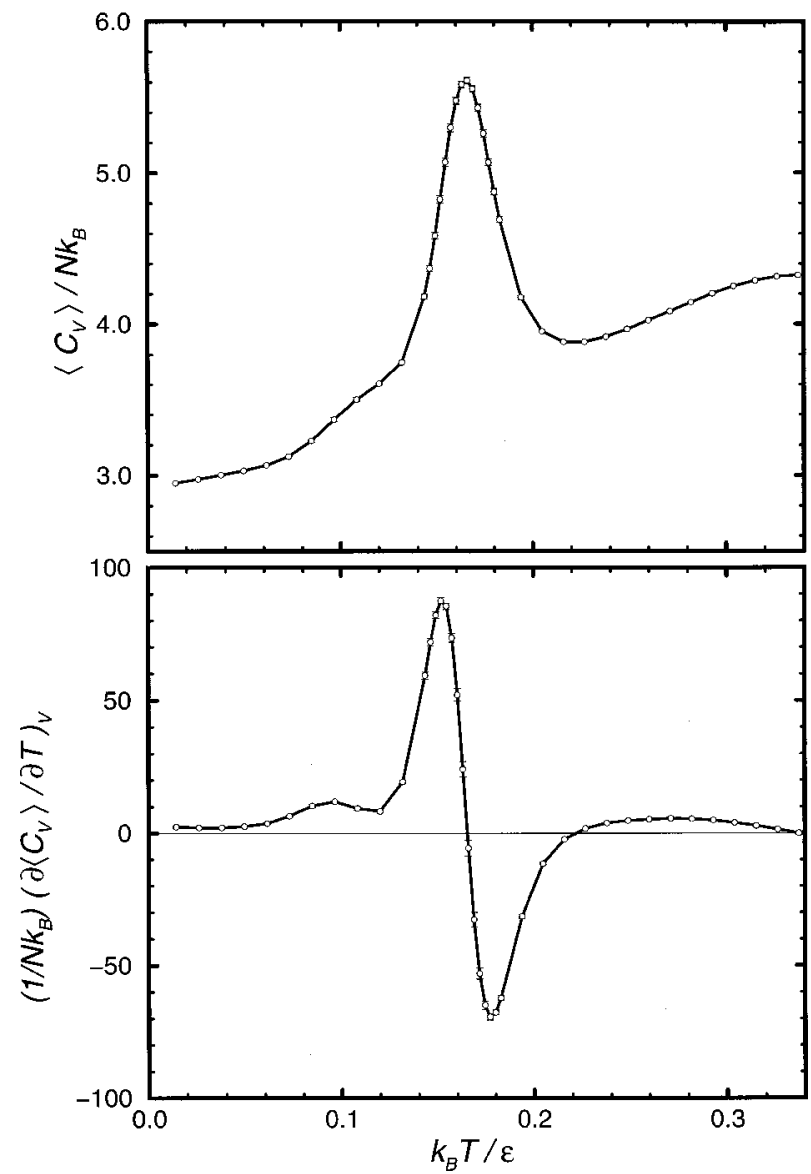

FIG. 1. The heat capacity $C_{V}$ per particle of $\mathrm{LJ}_{38}$ in units of $k_{B}$ (upper panel) and $\left(\partial C_{V} / \partial T\right)_{V}$ per particle (lower panel) as a function of reduced temperature. The small low temperature maximum in the derivative associated with a change in slope of the heat capacity identifies the transition region between the truncated octahedral basin and the icosahedral basin. The large heat capacity peak identifies the melting region.

ated using the procedure outlined above. In retrospect, we have found exchanges are only required between adjacent temperatures. We have also performed the calculations where exchanges are included only between adjacent temperatures, and we have seen no significant differences either in the final results or in the correlations between different temperatures. Using the random initializations of the clusters, after the initialization period the lowest temperature walks are dominated by configurations well represented by small amplitude oscillations about the global minimum structure.

For all data displayed in this work, the error bars represent two standard deviations of the mean. The heat capacity, calculated from the standard fluctuation expression of the energy

$$
C_{V}=k_{B} \beta^{2}\left[\left\langle E^{2}\right\rangle-\langle E\rangle^{2}\right],
$$

is displayed in the upper panel of Fig. 1. In agreement with the heat capacity for $\mathrm{LJ}_{38}$ reported by Doye et al. ${ }^{14}$ the heat capacity displayed in Fig. 1 has a melting maximum centered at about $T=0.166 \varepsilon / k_{B}$. In contrast to the results of Doye et al. ${ }^{14}$ we find no maximum associated with the solid-solid transition between the two basins in the potential surface. Rather, we see a small change in slope at about $T$
$=0.1 \varepsilon / k_{B}$. To characterize this region having a change in slope, in the lower panel of Fig. 1 we present a graph of $\left(\partial C_{V} / \partial T\right)_{V}$ calculated from the fluctuation expression

$$
\left(\frac{\partial C_{V}}{\partial T}\right)_{V}=-2 \frac{C_{V}}{T}+\frac{1}{k_{B}^{2} T^{4}}\left[\left\langle E^{3}\right\rangle+2\langle E\rangle^{3}-3\left\langle E^{2}\right\rangle\langle E\rangle\right] .
$$

The small low temperature maximum in $\left(\partial C_{V} / \partial T\right)_{V}$ occurs within the slope change region.

To interpret the configurations associated with the various regions of the heat capacity, we use an order parameter nearly identical to the order parameter introduced by Steinhardt, Nelson, and Ronchetti ${ }^{39}$ to distinguish face centered cubic from icosahedral structures in liquids and glasses. The order parameter has been used by Doye et al. ${ }^{5}$ to monitor phase changes in $\mathrm{LJ}_{38}$. The order parameter $Q_{4}$ is defined by the equation

$$
Q_{4}=\left(\frac{4 \pi}{9} \sum_{m=-4}^{4}\left|\bar{Q}_{4, m}\right|^{2}\right)^{1 / 2},
$$

where

$$
\bar{Q}_{4, m}=\frac{1}{N_{b}} \sum_{r_{i j}<r_{b}} Y_{4, m}\left(\theta_{i j}, \phi_{i j}\right) .
$$

To understand the parameters used in Eq. (17), it is helpful to explain how $\bar{Q}_{4, m}$ is evaluated. The center of mass of the full 38 atom cluster is located and the atom closest to the center of mass is then identified. The atom closest to the center of mass plus the 12 nearest neighbors of that atom define a "core" cluster of the 38 atom cluster. The center of mass of the core cluster is then calculated. The summation in Eq. (17) is performed over all vectors that point from the center of mass of the core cluster to all $N_{b}$ bonds formed from the 13 atoms of the core cluster. A bond is assumed to be formed between two atoms of the core cluster if their internuclear separation $r_{i j}$ is less than a cutoff parameter $r_{b}$, taken to be $r_{b}=1.39 \sigma$ in this work. In Eq. (17) $\theta_{i j}$ and $\phi_{i j}$ are, respectively, the polar and azimuthal angles of the vector that points from the center-of-mass of the core cluster to the center of each bond, and $Y_{4, m}(\theta, \phi)$ is a spherical harmonic. To verify that the optimal value of $Q_{4}$ is obtained, the procedure is repeated by choosing the second closest atom to the center of mass of the whole cluster to define the core cluster. The value of $Q_{4}$ obtained from this second core cluster is compared with that obtained from the first core cluster, and the smallest resulting value of $Q_{4}$ is taken to be the value of $Q_{4}$ for the entire cluster.

In the work of Steinhardt et al. ${ }^{39}$ fewer bonds are included in the summation appearing in Eq. (17) than in the current work. In the definition used by Steinhardt et al. ${ }^{39}$ the only bonds that contribute to the sum in Eq. (17) are those involving the central atom of the core cluster. In the definition used in this work, at low temperatures the sum includes all the bonds included by Steinhardt et al. ${ }^{39}$ in addition to vectors that connect the center of mass of the core cluster with the centers of bonds that connect atoms at the surface of the core cluster with each other. For a perfect and undistorted icosahedral or truncated octahedral cluster, the current definition and the definition of Steinhardt et al. ${ }^{39}$ are identical 


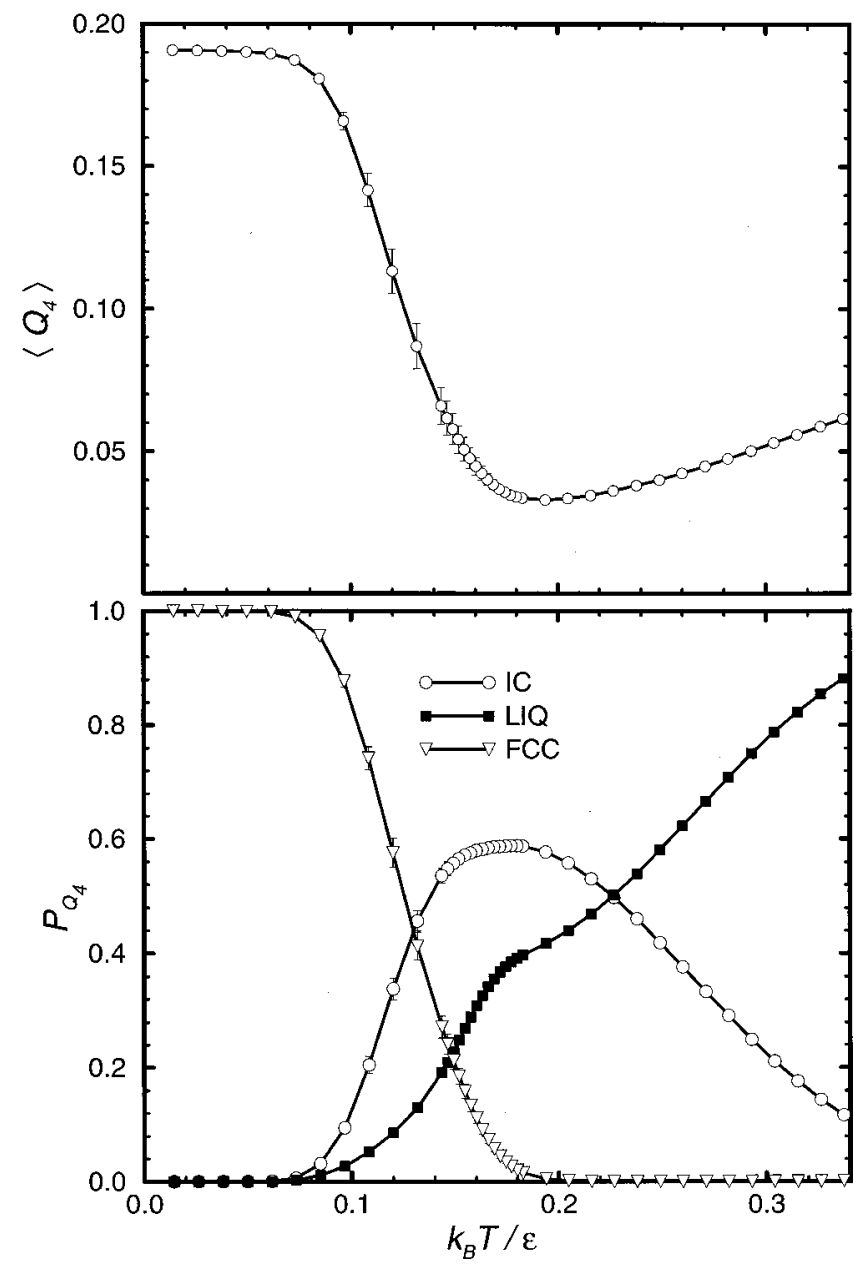

FIG. 2. The expectation value of the order parameter (upper panel) and the order parameter probability distribution (lower panel) as a function of reduced temperature. In the lower panel FCC labels the truncated octahedron, IC labels structures from the icosahedral basin and LIQ labels structures from the liquid region. The transition between FCC and IC occurs at the same temperature as the low temperature peak in $\left(\partial C_{V} / \partial T\right)_{V}$ in Fig. 1.

numerically owing to the rotational symmetry of the spherical harmonics. However, for distorted clusters the two definitions differ numerically. For perfect, undistorted icosahedral clusters $Q_{4}=0$ whereas for perfect, undistorted truncated octahedral clusters, $Q_{4} \cong 0.19$, and both definitions of the order parameter are able to distinguish configurations from the truncated octahedral basin and other basins at finite temperatures. However, we have found the definition introduced by Steinhardt et al. ${ }^{39}$ is unable to distinguish structures in the icosahedral basin from liquidlike structures. This same issue has been discussed previously by Lynden-Bell and Wales. ${ }^{40}$ In contrast, we have found that liquidlike structures have larger values of $Q_{4}$ than icosahedral structures when the present definition of $Q_{4}$ [i.e., the definition that includes additional bonds in Eq. (17)], is used. Consequently, as discussed shortly, the current definition of $Q_{4}$ enables an association of each configuration with either the icosahedral basin, the truncated octahedral basin, or structures that can be identified as liquidlike.

The average of $Q_{4}$ as a function of temperature is plotted in the upper panel of Fig. 2. Again the error bars represent two standard deviations of the mean. At the lowest calculated temperatures $\left\langle Q_{4}\right\rangle$ is characteristic of the global truncated octahedral minimum. As the temperature is raised to the point where the slope change begins in the heat capacity, $\left\langle Q_{4}\right\rangle$ begins to drop rapidly signifying the onset of transitions between the structures associated with the global minimum and icosahedral structures. We then have the first hint that the slope change in $C_{V}$ is associated with an analogue of a solid-solid transition from the truncated octahedron to icosahedral structures.

To clarify the transition further, the data plotted in the lower panel of Fig. 2 represent the probability of observing particular values of $Q_{4}$ as a function of temperature. The probabilities have been calculated by tabulating the frequency of observing particular values of $Q_{4}$ for each configuration generated in the simulation. Different values of $Q_{4}$ are then assigned to either icosahedral structures (labeled IC in the graph), truncated octahedral structures (labeled FCC) or liquidlike structures (labeled LIQ). By comparing the lower panel of Fig. 2 with the derivative of the heat capacity plotted in the lower panel of Fig. 1, it is evident that icosahedral structures begin to be occupied and the probability of finding truncated octahedral structures begins to fall when the derivative in the heat capacity begins to rise. Equilibrium between the truncated octahedral structures and the icosahedral structures continues into the melting region, and truncated octahedral structures only disappear on the high temperature side of the melting peak of the heat capacity. Doye et al. ${ }^{14}$ and Miller et al. ${ }^{15}$ have generated data analogous to that depicted in the lower panel of Fig. 2 using the superposition method, and the data of Miller et al. ${ }^{15}$ are in qualitative agreement with the present data. A more direct comparison with the data of these authors can be made by performing periodic quenching along the parallel tempering trajectories. We then use an energy criterion similar to that of Doye et al. ${ }^{14}$ to distinguish the three categories of geometries and to generate the respective probabilities $P$. For a given total cluster energy $E$, a truncated octahedron is associated with $E<-173.26 \varepsilon$, icosahedral-based structures with $-173.26 \varepsilon \leqslant E<-171.6 \varepsilon$, and liquidlike structures with $E$ $\geqslant-171.6 \varepsilon$. The quenches have been performed every $10^{4}$ MC steps for each temperature, and the results of these quenches are plotted in Fig. 3. Using the energy criterion, the behavior we observe is qualitatively similar to the data of Doye et al. ${ }^{14}$ However, the largest probability of observing icosahedral structures is found here to be substantially lower than Doye et al. ${ }^{14}$ The data accumulated more recently by Miller et al. ${ }^{15}$ using the superposition method include contributions from more stationary points than in the previous work of Doye et al., ${ }^{14}$ but no reweighting has been performed. As a result, the distributions of isomers look quite different, especially at high temperatures. ${ }^{14}$

The assignment of a particular value of $Q_{4}$ to a structure as displayed in Fig. 2, is made by an analysis of the probability distribution $P_{Q}\left(T, Q_{4}\right)$ of the order parameter displayed in Figs. 4 and 5. Figure 4 is a representation of the three-dimensional surface of $P_{Q}\left(T, Q_{4}\right)$ as a function of temperature and order parameter. A projection of this surface onto two dimensions is given in Fig. 5. The probability den- 


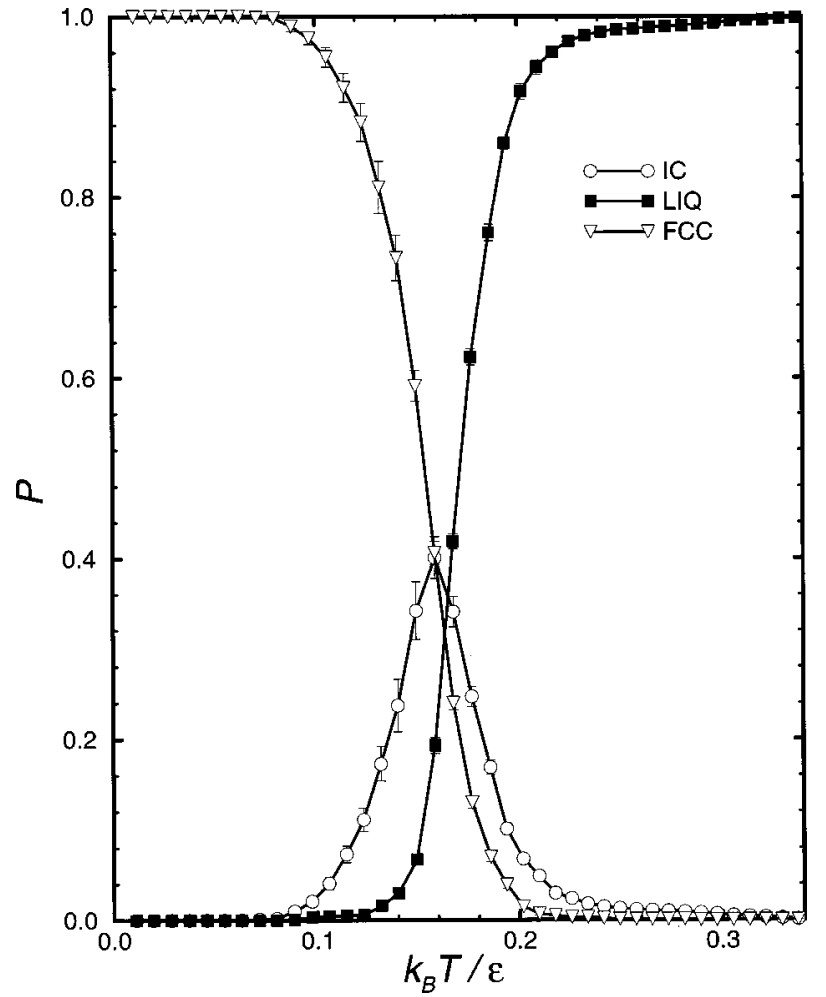

FIG. 3. The probability distributions of observing different structures as a function of temperature using the energy criterion. The labels are the same as those defined in the lower panel of Fig. 2, and the data complements the interpretation of the lower panel of Fig. 2

sity in Fig. 5 is represented by the shading so that the brighter the area the greater the probability. The horizontal white lines in Fig. 5 define the regions of the heat capacity curve. The lowest temperature horizontal line represents the temperature at which the slope of the heat capacity first changes rapidly, the middle temperature horizontal line represents the lowest temperature of the melting peak and the highest temperature horizontal line represents the end of the melting region. An additional representation of the data is given in Fig. 6, where the probability of observing particular values of $Q_{4}$ is given as a function of $Q_{4}$ at a fixed tempera-

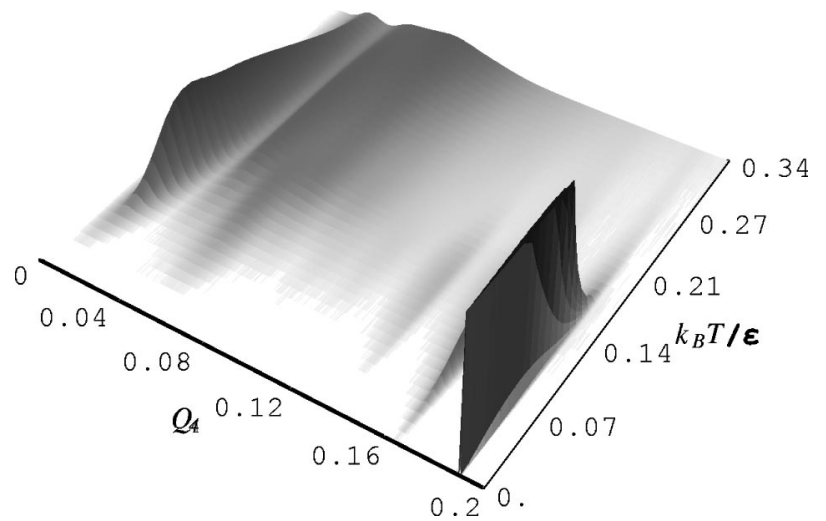

FIG. 4. The probability of observing configurations with particular values of $Q_{4}$ with $Q_{4}$ displayed along one axis and the reduced temperature displayed along the other axis. The large peak at low temperatures comes from the truncated octahedral structures and the broad region with small $Q_{4}$ at intermediate temperatures represents structures in the icosahedral basin.

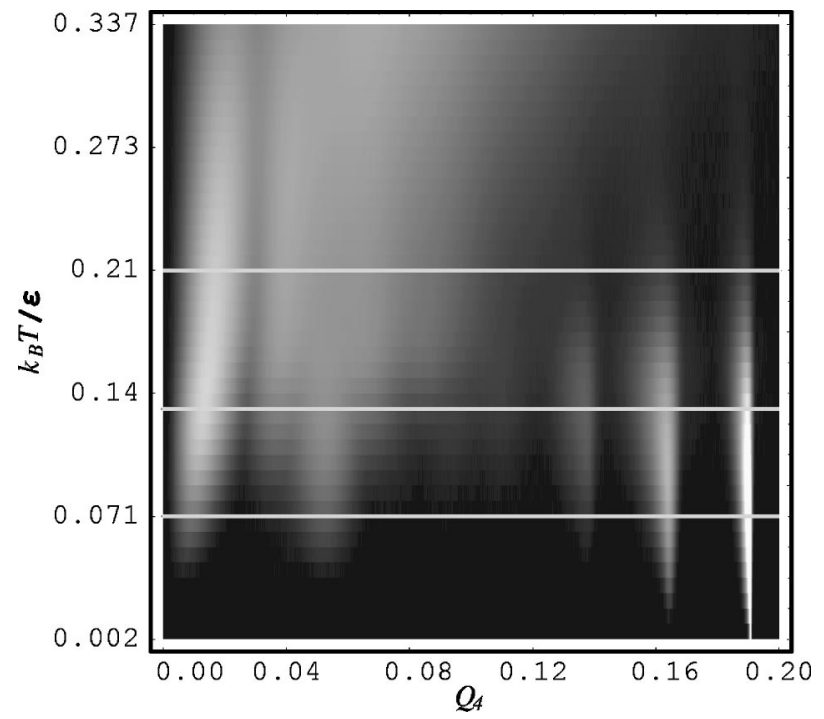

FIG. 5. A projection of Fig. 4 onto the $T$ - $Q_{4}$ plane. The probability is measured by the shading with increasing probability represented by lighter shading. The lowest temperature horizontal white line represents the temperature at which transitions between the icosahedral and lowest energy basins begin. The second lowest temperature horizontal white line represents the beginning of the melting region, and the highest temperature horizontal white line represents the end of the melting peak of the heat capacity. The coexistence of icosahedral and octahedral structures continues into the melting region.

ture of $0.14 \varepsilon / k_{B}$. In Fig. 6 three regions are evident for $P_{Q}\left(T=0.14 \varepsilon / k_{B}, Q_{4}\right)$ with $Q_{4}$ ranging from 0.13 to 0.19 . Although the presence of three regions seems to indicate three distinct structures, all three regions correspond to the truncated octahedral global minimum. We have verified this assignment by quenching the structures with $Q_{4}$ ranging from 0.13 to 0.19 to their nearest local minima, and we have found all such structures quench to the truncated octahedron. To explain the three regions, we have found that there are small distortions of $\mathrm{LJ}_{38}$ about the truncated octahedral structure where both the energy and $Q_{4}$ increase together. These regions where both the energy and $Q_{4}$ increase above $Q_{4}$ $\cong 0.13$ have low probability and account for the oscillations observed in Figs. 4-6. In the lower panel of Fig. 2, all structures having $Q_{4}>0.13$ have been identified as truncated octahedra. Quench studies of the broad region visible in Fig. 5 at the lowest values of $Q_{4}$, or equivalently in the first low $Q_{4}$ peak in Fig. 6 find all examined structures to belong to the icosahedral basin. To determine if a given configuration is associated with the icosahedral basin, one-dimensional cross-sectional plots are made from Fig. 4 at each temperature used in the calculation. Figure 6 is a particular example of such a cross-sectional plot. The maximum present at low $Q_{4}$ represents the center for structures in the icosahedral ba$\sin$. The next two maxima at higher $Q_{4}$ represents the midpoint of the liquid region. Consequently, in generating the lower panel of Fig. 2, all configurations with $Q_{4}$ between $Q_{4}=0$ and the first minimum in Fig. 6 have been identified as icosahedral structures. All other values of $Q_{4}$, represented by the broad intermediate band in Fig. 5 (or the region about the second two maxima in Fig. 6), have been identified as liquidlike structures. To make these identifications, separate 


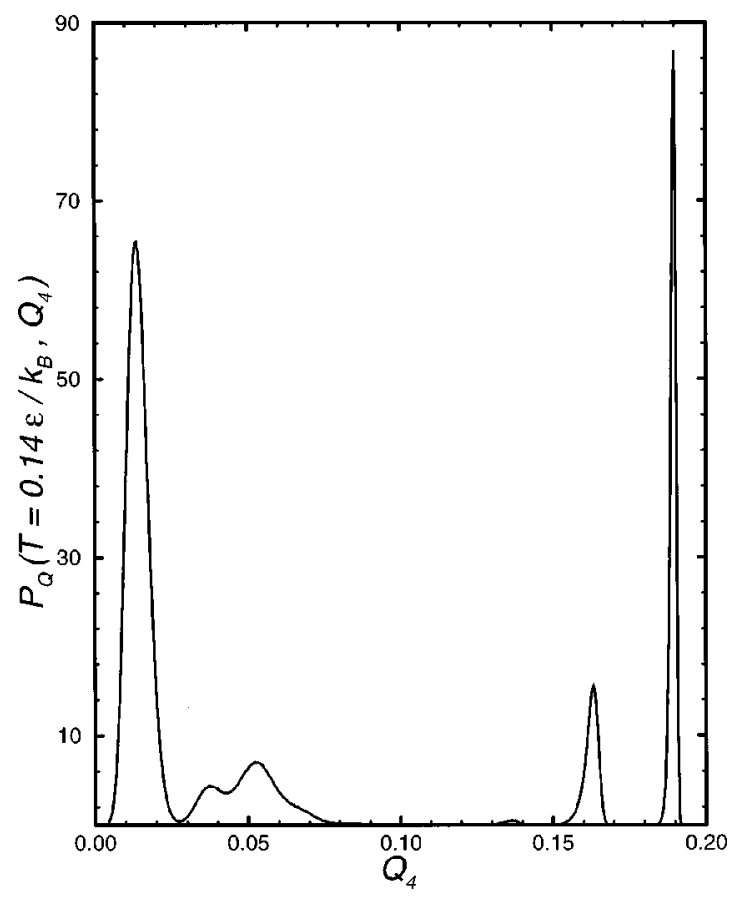

FIG. 6. The probability of observing configurations with particular values of $Q_{4}$ as a function of $Q_{4}$ at $T=0.14 \varepsilon / k_{B}$. The region from $Q_{4}=0$ through the first maximum to the first minimum defines the icosahedral basin at $T$ $=0.14 \varepsilon / k_{B}$, the region from the first minimum to the third defines liquidlike structures, and the region about the three maxima having the highest values of $Q_{4}$ define the truncated octahedral basin. The oscillations in the truncated octahedral basin arise from distorted structures of low probability where both the energy and $Q_{4}$ rise together.

cross sections of Fig. 4 must be made at each temperature. Of course, it is impossible to verify that the identification of all values of $Q_{4}$ with a particular structure as discussed above would agree with the result of quenching the structure to its nearest potential minimum. The differences found by defining icosahedral, truncated octahedral or liquidlike structures using either an energy criterion or $Q_{4}$ is clarified by comparing Fig. 3 and the lower panel of Fig. 2. Both definitions are arbitrary, and the information carried by the two classification methods complement each other.

Figure 5 also provides additional evidence that the peak in $\left(\partial C_{V} / \partial T\right)_{V}$ is associated with the equilibrium between the truncated octahedral structures and the icosahedral structures. There is significant density for both kinds of structures in the region between the lowest two parallel lines that define the region with the slope change. Additionally, both icosahedral structures and truncated octahedral structures begin to be in equilibrium with each other at the beginning of the slope change region. This equilibrium continues to temperatures above the melting region.

Another identification of the slope change region with a transition between truncated octahedral and icosahedral forms can be made by defining $P_{R}(T, R) d R$ to be the probability that an atom in the cluster is found at location $R$ to $R+d R$ from the center-of-mass of the cluster at temperature $T$. A projection of $P_{R}(T, R)$ onto the $R$ and $T$ plane is depicted in Fig. 7. The solid vertical lines represent the location of atoms from the center-of-mass of the truncated octahedral structure (the lower set of vertical lines), and the lowest en-

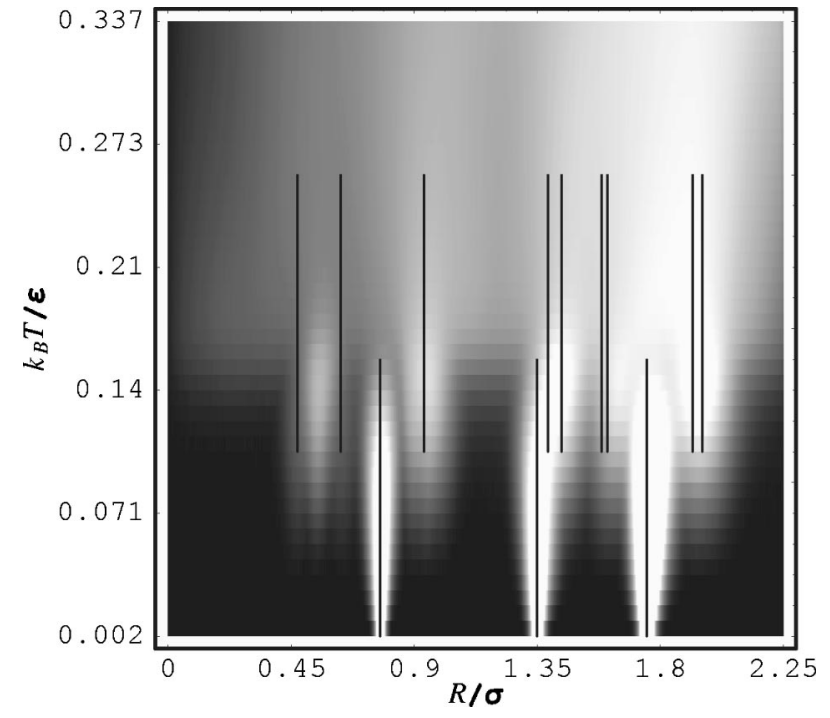

FIG. 7. The projected probability of observing particles at distance $R$ from the center-of-mass of $\mathrm{LJ}_{38}$ as a function of $R$ and reduced temperature. As in Fig. 5 increased probability is represented by the lightest shading. The lower vertical lines represent the location of atoms in the fully relaxed truncated octahedron and the upper vertical lines represent the location of atoms in the fully relaxed icosahedral structure that is lowest in energy. Equilibrium between the icosahedral and octahedral forms are observed in the same temperature range as found in Figs. 2 and 5.

ergy icosahedral structure (the upper set of vertical lines). As in Fig. 5, increased probability is represented by the lighter shading. At the lowest temperatures $P_{R}(T, R)$ is dominated by contributions from the truncated octahedron as is evident by comparing the shaded regions with the lowest set of vertical lines. As the temperature is increased, contributions to $P_{R}(T, R)$ begin to appear from the icosahedral structures. The shaded region at $R=0.45$ does not match any of the vertical lines shown, but corresponds to atoms in the third lowest energy isomer, which like the second lowest energy isomer, comes from the icosahedral basin. The equilibrium between the icosahedral and truncated octahedral structures observed in Fig. 7 matches the regions of temperature observed in Fig. 5.

We have mentioned previously that parallel tempering introduces correlations in the data accumulated at different temperatures, and it is important to ensure the statistical errors are sufficiently small that observed features are real and not artifacts of the correlations. To measure these correlations we define a cross temperature correlation function for some temperature dependent property $g$ by

$\gamma\left(T_{1}, T_{2}\right)=\frac{\left\langle\left(g\left(T_{1}\right)-\left\langle g\left(T_{1}\right)\right\rangle\right)\left(g\left(T_{2}\right)-\left\langle g\left(T_{2}\right)\right\rangle\right)\right\rangle}{\left[\left\langle\left(g\left(T_{1}\right)-\left\langle g\left(T_{1}\right)\right\rangle\right)^{2}\right\rangle\left\langle\left(g\left(T_{2}\right)-\left\langle g\left(T_{2}\right)\right\rangle\right)^{2}\right\rangle\right]^{1 / 2}}$.

A projection of $\gamma\left(T_{1}, T_{2}\right)$ when $g=C_{V}$ is given in Fig. 8. In Fig. 8 white represents $\gamma=1$ and black represents $\gamma=-1$ with other shadings representing values of $\gamma$ between these two extremes. The white diagonal line from the lower left hand corner to the upper right hand corner represents the case that $T_{1}=T_{2}$ so that $\gamma=1$. The light shaded areas near this diagonal represent cases where $T_{1}$ and $T_{2}$ are adjacent temperatures in the parallel tempering simulations, and we 


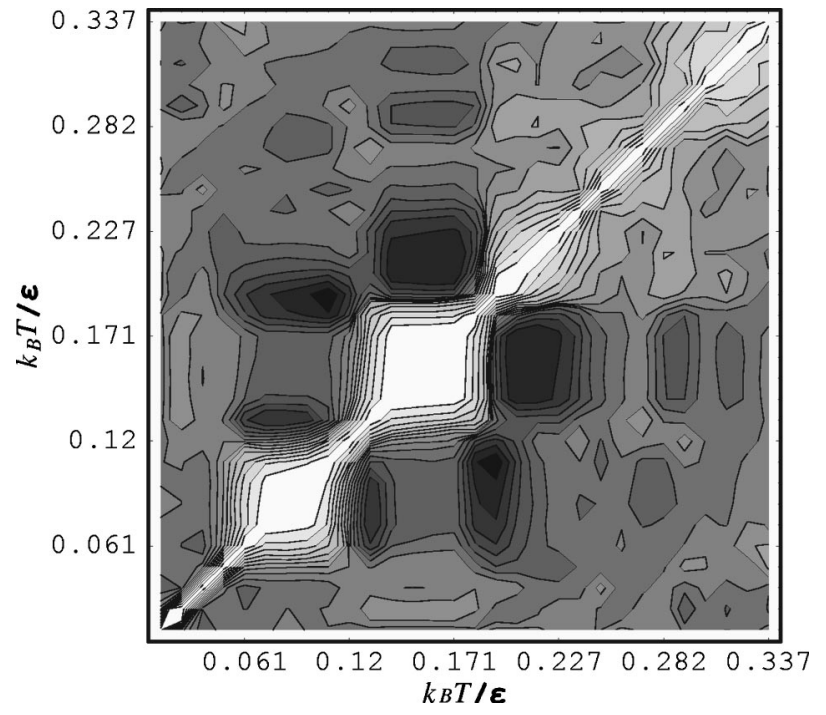

FIG. 8. $\gamma\left(T_{1}, T_{2}\right)$ for the heat capacity as defined in Eq. (18) (with $g$ $=C_{V}$ ) as a function of reduced temperature along two axes. White shading represents $\gamma=1$ and black represents $\gamma=-1$. The white diagonal line connecting the lower left-hand corner with the upper right-hand corner indicates $T_{1}=T_{2}$ so that $\gamma=1$. The black areas show anticorrelation from parallel tempering between the heat capacity calculated at the maximum of the heat capacity and the center of the change in slope region.

find $\gamma$ to be only slightly less than unity. More striking are the black regions off the diagonal where $\gamma$ is nearly -1 . These black regions correspond to anti-correlations between results at temperatures near the heat capacity maximum in the melting peak and temperatures near the center of the slope change region associated with the transition between icosahedral and truncated octahedral structures. These correlations imply the importance of performing sufficiently long simulations to ensure that statistical fluctuations of the data are small compared to important features in the data as a function of temperature.

\section{CONCLUSIONS}

Using parallel tempering methods we have successfully performed ergodic simulations of the equilibrium thermodynamic properties of $\mathrm{LJ}_{38}$ in the canonical ensemble. As discussed by Doye et al..$^{5}$ the potential surface of this system is complex with two significant basins; a narrow basin about the global minimum truncated octahedral structure, and a wide icosahedral basin. These two basins are separated both by structure and a large energy barrier making simulations difficult. In agreement with the results of Doye et al. ${ }^{14}$ we find clear evidence of equilibria between structures at the basin of the global minimum and the icosahedral basin at temperatures below the melting region. Unlike previous work we find no heat capacity maximum associated with this transition, but rather a region with a change in the slope of the heat capacity as a function of temperature.

We have found parallel tempering to be successful with this system, and have noted correlations in our data at different temperatures when the parallel tempering method is used. These correlations imply the need to perform long simulations so that the statistical errors are sufficiently small that the correlations do not introduce artificial conclusions.
We believe that the methods used in this work could be applied to a variety of other systems including clusters of complexity comparable to $\mathrm{LJ}_{38}$. For instance, the 75-atom Lennard-Jones cluster is known to share many features with the 38 -atom cluster investigated here. $\mathrm{LJ}_{75}$ is also characterized by a double funnel energy landscape, one funnel being associated with icosahedral structures, and the other funnel being associated with the decahedral global minimum. The landscape of $\mathrm{LJ}_{75}$ has been recently investigated by Doye, Miller, and Wales ${ }^{6}$ who have used $Q_{6}$ as the order parameter. In another paper, ${ }^{2}$ Wales and Doye have predicted that the temperature where the decahedral/icosahedral equilibrium takes place should be close to $0.09 \varepsilon / k_{B}$. This prediction is made by using the superposition method, but no caloric curves have yet been reported for $\mathrm{LJ}_{75}$. The parallel tempering Monte Carlo method can be expected to work well for $\mathrm{LJ}_{75}$, and such a parallel tempering study would be another good test case for theoretical methods discussed in this work.

A useful enhancement of parallel tempering Monte Carlo is the use of multiple histogram methods ${ }^{13,41}$ that enables the calculation of thermodynamic functions in both the canonical and microcanonical ensembles by the calculation of the microcanonical entropy. In practice the multiple histogram method requires the generation of histograms of the potential energy at a set of temperatures such that there is appreciable overlap of the potential energy distributions at adjacent temperatures. This overlap requirement is identical to the choice of temperatures needed in parallel tempering.

In performing simulations on $\mathrm{LJ}_{38}$ we have tried other methods to reduce ergodicity errors, and we close this section by summarizing the difficulties we have encountered with these alternate methods. It is important to recognize that the parallel tempering simulations include in excess of $10^{10}$ Monte Carlo points, and most of our experience with these alternate methods have come from significantly shorter simulations. Our ability to include this large number of Monte Carlo points with parallel tempering is an important reason why we feel parallel tempering is so useful.

From experience with other smaller and simpler clusters, for a J-walking simulation to include $10^{10}$ points, an external distribution containing at least $10^{9}$ points is required to prevent oversampling of the distribution. Such a large distribution is prohibitive with current computer technology. Our J-walking simulations containing about $10^{7}$ Monte Carlo points have resulted in data that have not been internally reproducible, and data that are not in good agreement with the parallel tempering data. Many long J-walking simulations with configurations initiated at random only have icosahedral structures at the lowest calculated temperatures. To stabilize the J-walking method with respect to the inclusion of truncated octahedral structures at low temperatures, we have attempted to generate distributions using the modified potential energy function $U_{m}(\mathbf{r}, \lambda)=U(\mathbf{r})-\lambda Q_{4}$. In this modified potential $\lambda$ is a parameter chosen to deepen the octahedral basin without significantly distorting the cluster. While this modified potential has led to more stable results than J-walking using the bare potential, the results with $10^{8}$ Monte Carlo points have not been reproducible in detail. The 
application of Tsallis distributions ${ }^{24}$ has not improved this situation.

We have also tried to apply the multicanonical J-walking approach recently introduced by Xu and Berne. ${ }^{25}$ While this multicanonical approach has been shown to improve the original J-walking strategy for other cluster systems, in the case of $\mathrm{LJ}_{38}$ the iterations needed to produce the external multicanonical distribution have not produced truncated octahedral structures. The iterations have produced external distributions having either liquidlike structures or structures from the icosahedral basin. The multicanonical distribution is known to have deficiencies at low energies, and this low energy difficulty appears to be problematic for $\mathrm{LJ}_{38}$. We have attempted to solve these deficiencies by including prior information about the thermodynamics of the system. In this attempt we have chosen the multicanonical weight to be $w_{\mathrm{mu}}(U)=\exp \left[-S_{\mathrm{PT}}(U)\right]$ where $S_{\mathrm{PT}}(U)$ is the microcanonical entropy extracted from a multihistogram analysis ${ }^{13,41}$ of a parallel tempering Monte Carlo simulation. In several attempts using this approach we have not observed either the truncated octahedral structure nor structures from the icosahedral basin with significant probability. The multicanonical distribution so generated is dominated by liquidlike structures, and the distribution appears to be incapable of capturing the solid-to-solid transition that leads to the low temperature peak in $\left(\partial C_{V} / \partial T\right)_{V}$. Whether there are other approaches to generate a multicanonical distribution that are more successful in capturing low temperature behaviors is unknown to us.

Much insight about phase change behaviors can be obtained from simulations in the microcanonical ensemble or using molecular dynamics methods. For example, the van der Waals loops observed in $\mathrm{LJ}_{55}$ (Ref. 13) complement the interpretation of the canonical caloric curves. In the next paper ${ }^{27}$ we present parallel tempering results for $\mathrm{LJ}_{38}$ using both molecular dynamics and microcanonical Monte Carlo methods.

\section{ACKNOWLEDGMENTS}

Some of this work has been motivated by the attendance of two of us (D.L.F. and F.C.) at a recent CECAM meeting on "Overcoming broken ergodicity in simulations of condensed matter systems." We would like to thank CECAM, J.E. Straub, and B. Smit who organized the meeting, and those who attended the workshop for stimulating discussions, particularly on the connections between J-walking and parallel tempering. Two of us (D.L.F. and J.P.N.) would also like to thank Professor M.P. Nightingale for helpful discussions concerning the parallel tempering method. This work has been supported in part by the National Science Foundation under Grant Nos. CHE-9714970 and CDA-9724347. This research has been supported in part by the Phillips Laboratory, Air Force Material Command, USAF, through the use of the MHPCC under cooperative agreement No. F29601-93-0001. The views and conclusions contained in this document are those of the authors and should not be interpreted as necessarily representing the official policies or endorsements, either expressed or implied, of Phillips Laboratory or the U.S. Government.

${ }^{1}$ F. F. Abraham, Homogeneous Nucleation Theory (Academic, New York, 1974).

${ }^{2}$ D. J. Wales and J. P. K. Doye, J. Phys. Chem. A 101, 5111 (1997).

${ }^{3}$ D. L. Freeman and J. D. Doll, Annu. Rev. Phys. Chem. 47, 43 (1996).

${ }^{4}$ J. Xie, J. A. Northby, D. L. Freeman, and J. D. Doll, J. Chem. Phys. 91, 612 (1989).

${ }^{5}$ J. P. K. Doye, M. A. Miller, and D. J. Wales, J. Chem. Phys. 110, 6896 (1999)

${ }^{6}$ J. P. K. Doye, M. A. Miller, and D. J. Wales, J. Chem. Phys. 111, 8417 (1999).

${ }^{7}$ R. H. Leary and J. P. K. Doye, Phys. Rev. E 60, R6320 (1999).

${ }^{8}$ R. Czerminski and R. Elber, J. Chem. Phys. 92, 5580 (1990).

${ }^{9}$ O. M. Becker and M. Karplus, J. Chem. Phys. 106, 1495 (1997).

${ }^{10}$ C. Kittel, Introduction to Solid State Physics, 4th ed. (Wiley, New York, 1971).

${ }^{11}$ E. K. Parks, G. C. Nieman, K. P. Kerns, and S. J. Riley, J. Chem. Phys. 107, 1861 (1997)

${ }^{12}$ R. S. Berry, T. L. Beck, H. L. Davis, and J. Jellinek, Adv. Chem. Phys. 70B, 75 (1988)

${ }^{13}$ P. Labastie and R. L. Whetten, Phys. Rev. Lett. 65, 1567 (1990).

${ }^{14}$ J. P. K. Doye, D. J. Wales, and M. A. Miller, J. Chem. Phys. 109, 8143 (1998).

${ }^{15}$ M. A. Miller, J. P. K. Doye, and D. J. Wales, Phys. Rev. E 60, 3701 (1999).

${ }^{16}$ D. J. Wales, Mol. Phys. 78, 151 (1993).

${ }^{17}$ J. P. K. Doye and D. J. Wales, J. Chem. Phys. 102, 9659 (1995).

${ }^{18}$ D. D. Frantz, D. L. Freeman, and J. D. Doll, J. Chem. Phys. 93, 2769 (1990).

${ }^{19}$ E. Marinari and G. Parisi, Europhys. Lett. 19, 451 (1992).

${ }^{20}$ C. J. Geyer and E. A. Thompson, J. Am. Stat. Assoc. 90, 909 (1995).

${ }^{21}$ M. C. Tesi, E. J. Janse van Rensburg, E. Orlandini, and S. G. Whittington, J. Stat. Phys. 82, 155 (1996).

${ }^{22}$ M. Falcioni and M. W. Deem, J. Chem. Phys. 110, 1754 (1999).

${ }^{23}$ Q. Yan and J. J. de Pablo, J. Chem. Phys. 111, 9509 (1999).

${ }^{24}$ I. Andricioaei and J. E. Straub, J. Chem. Phys. 107, 9117 (1997).

${ }^{25}$ H. Xu and B. J. Berne, J. Chem. Phys. 110, 10299 (1999).

${ }^{26}$ B. Berg and T. Neuhaus, Phys. Rev. Lett. 68, 9 (1991).

${ }^{27}$ F. Calvo, J. P. Neirotti, D. L. Freeman, and J. D. Doll, J. Chem. Phys. 112, 10350 (2000), following paper.

${ }^{28}$ M. A. Kalos and P. A Whitlock, Monte Carlo Methods (Wiley, New York, 1986).

${ }^{29}$ D. Frenkel and B. Smit, Understanding Molecular Simulations (Academic, San Diego, 1996).

${ }^{30}$ N. Metropolis, A. W. Rosenbluth, M. N. Rosenbluth, A. H. Teller, and E. Teller, J. Chem. Phys. 21, 1087 (1953).

${ }^{31}$ A. Matro, D. L. Freeman, and R. Q. Topper, J. Chem. Phys. 104, 8690 (1996).

${ }^{32}$ A. Dullweber, M. P. Hodges, and D. J. Wales, J. Chem. Phys. 106, 1530 (1997).

${ }^{33}$ D. D. Frantz, D. L. Freeman, and J. D. Doll, J. Chem. Phys. 97, 5713 (1992).

${ }^{34}$ M. A. Strozak, G. E. Lopez, and D. L. Freeman, J. Chem. Phys. 97, 4445 (1992).

${ }^{35}$ G. E. Lopez and D. L. Freeman, J. Chem. Phys. 98, 1428 (1993).

${ }^{36}$ E. Curotto, D. L. Freeman, B. Chen, and J. D. Doll, Chem. Phys. Lett. 295, 366 (1998).

${ }^{37}$ C. J. Tsai and K. D. Jordan, J. Chem. Phys. 99, 6957 (1993).

${ }^{38}$ A. J. Acevedo, L. M. Caballero, and G. E. Lopez, J. Chem. Phys. 106, 7257 (1997).

${ }^{39}$ P. J. Steinhardt, D. R. Nelson, and M. Ronchetti, Phys. Rev. B 28, 784 (1983).

${ }^{40}$ R. M. Lynden-Bell and D. J. Wales, J. Chem. Phys. 101, 1460 (1994).

${ }^{41}$ A. M. Ferrenberg and R. H. Swendsen, Phys. Rev. Lett. 61, 2635 (1988). 